\title{
Application of Matter Element Analysis in Power Grid Development Diagnosis
}

\author{
Chao $\mathrm{Xu}^{1}$, Di Huang ${ }^{1}$, Zhe Chen ${ }^{2}$, Dong Peng ${ }^{3}$, Lang Zhao ${ }^{3, \mathrm{a}}$ and Tianqi Zhang ${ }^{3}$ \\ ${ }^{1}$ State Grid Jiangsu Electric Power Co., Ltd., 210000 Jiangsu, China \\ ${ }^{2}$ State Grid Jiangsu Electric Power Co., Ltd., Electric Power Research Institute, 210000 Jiangsu, China \\ ${ }^{3}$ State Grid Economic and Technological Research Institute Co., Ltd, 102209 Beijing, China
}

\begin{abstract}
The structure and form of modern power grids are becoming more and more complex, and there are many indicators and factors that need to be considered to evaluate the development status of the power grid. In order to diagnose the development level of power grid, the index system is reconstructed, which includes seven dimensions: the coordination of power grid development speed, the coordination of power grid development scale, the coordination of power grid structure, the security and reliability of power grid, the level of equipment, the utilization efficiency of power grid and the development benefit of power grid, the construction principle and index selection basis are given. When dealing with contradictory problems in reality, methods combining quantitative and qualitative can be applied to study. The matter element analysis method is applied to power grid development diagnosis, and a model of power grid development diagnosis based on matter element analysis is established, the model can not only diagnose the development level of power grid, but also determine the key factors that affect the diagnosis results, and give suggestions for improvement. The practical example verifies the feasibility and rationality of the method, which has good evaluation accuracy and prediction effect.
\end{abstract}

\section{Introduction}

With the rapid development of national economy, the scale of power grid continues to expand, the structure of $\mathrm{AC} / \mathrm{DC}$ hybrid is more and more complex [1], and all kinds of problems restricting the development of power grid are exposed one after another [2]. In such a situation, it has become an important work to evaluate the development level of power grid, find out the short board in the process of power grid development, and provide the basis for planning power grid development and making investment decisions.

At present, the research of power grid development diagnosis has achieved some results, but the relevant system index is not comprehensive enough, and the current research methods of power grid development diagnosis mainly focus on the fuzzy analysis method. By calculating the membership degree of each index, the relationship between the quantity to be evaluated and the grade interval is quantified by using the principle of fuzzy mathematics, but the fuzzy evaluation cannot deal with the problem of the same membership degree.

In order to solve such contradictory problems in reality, some scholars proposed matter element analysis method [3], which expanded the evaluation interval from to, that is, the whole real number axis, to realize the fine description of the difference problem in the level interval, thus solving the problem of approximate membership [4], and using the characteristic that the correlation value can be negative to dynamically describe the change trend of things. This advantage of matter element analysis makes it applied in many fields [5-6].

Based on the existing diagnosis index system of power grid development, this paper reconstructs the index system of seven dimensions, including the coordination of power grid development speed, the coordination of power grid development scale, the coordination of power grid structure, the safety and reliability of power grid, the level of equipment, the utilization efficiency of power grid, and the development benefit of power grid, which can reflect the development level of power grid. The construction principle and index selection basis are given. By applying the concept of matter element model, the relationship between each index and evaluation grade is quantified. Combined with the operation criteria of power grid and the sensitivity of power grid development level to different indicators, analytic hierarchy process (AHP) 1-9 scaling method is used to determine the weight, and a diagnosis model of power grid development based on matter element analysis is established. The effectiveness of the method in diagnosing the development level of power grid is verified by an example. According to the classification results and transformation trend of single index, the key factors affecting the development level of power grid are determined, and the development suggestions are put forward.

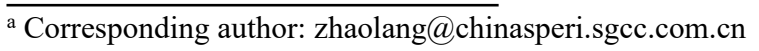




\section{Matter element analysis model in power grid development diagnosis}

\subsection{Principles of matter element analysis}

Matter element analysis method is a research method that combines qualitative and quantitative analysis to solve the contradiction problems in reality. The method of matter element analysis constructs the ternary ordered combination of name, feature and value to describe the quantity value of a feature. Taking matter element as basic element, the relationship between point and evaluation interval is described as distance, evaluation interval and qualitative range interval constitute interval set, and the relationship between point and interval set is defined as position value. This paper studies the calculation formula of correlation function, objectively characterizes the degree of certain properties of things, and quantitatively describes the changes of properties of things. The calculation of correlation function does not need to rely on subjective judgment or statistics, but is determined according to the required range and the interval of qualitative change of a feature, so that the correlation function can get rid of the deviation caused by subjective judgment.

Matter element analysis method comprehensively considers the situation that things belong to the inside and outside of the set, and extends the evaluation interval of traditional analysis method to positive value, which more precisely describes the degree of things belonging to the evaluation interval, solves the problem of similar membership degree, and negative value reflects the possibility of things transforming into the evaluation interval, so as to obtain the development level and change trend of the research object.

\subsection{Diagnosis of power grid development}

According to the establishment principle of index system, the development level of power grid is divided into seven dimensions: the coordination of power grid development speed, the coordination of power grid development scale, the coordination of power grid structure, the safety and reliability of power grid, the level of equipment, the utilization efficiency of power grid and the development benefit of power grid. Each dimension influences and restricts each other.

In order to quantitatively and qualitatively analyse the development level of power grid, it is necessary to quantify the relationship between a single index and the evaluation interval in the dimension index system. In this paper, the matter element model and correlation function in matter element analysis are used.

The matter element model describes the object of study with the given name $N$, characteristic $c$ and its value $v$. The ordered triple $R=(N, c, v)$ is the basic element to describe things. Things with multiple characteristics $c_{1}, c_{2} \ldots c_{\mathrm{n}}$ can be described by the corresponding value $v_{1}, v_{2} \ldots v_{\mathrm{n}}$, expressed as

$$
R=\left[\begin{array}{ccc}
N & c_{1} & v_{1} \\
& c_{2} & v_{2} \\
& \cdots & \cdots \\
& c_{n} & v_{n}
\end{array}\right]
$$

The evaluation results of each index are divided into five grades, excellent, good, medium, qualified and poor.

The node domain matter element represents the value range of all features.

$$
R_{p}=\left[\begin{array}{ccc}
N & c_{1} & \left(c_{1}, d_{1}\right) \\
& c_{2} & \left(c_{2}, d_{2}\right) \\
& \cdots & \cdots \\
& c_{n} & \left(c_{n}, d_{n}\right)
\end{array}\right]
$$

where $N$ is the development level of all levels of power grid, $c_{1}-c_{n}$ is the index of all levels, $\left(c_{n}, d_{n}\right)$ is the value range of $c_{n}$ under the development level of all levels of power grid.

The object element to be evaluated represents the measurement data of each index to be evaluated, which is expressed as:

$$
R=\left[\begin{array}{lll}
N_{0} & c_{1} & x_{1} \\
& c_{2} & x_{2} \\
& \cdots & \cdots \\
& c_{n} & x_{n}
\end{array}\right]
$$

where $N_{0}$ is the development level of power grid to be evaluated, $c_{1}-c_{n}$ is the index of the power grid level to be evaluated, $x_{n}$ is the measurement of $c_{n}$.

\subsection{Determination of the index layer correlation degree}

After determining the classical domain matter element, nodal domain matter element and to be evaluated matter element, the correlation function can be used to quantify the similarity degree between the to be evaluated matter element and the classical domain matter element and nodal domain matter element.

When $X_{0}=(a, b), X=(c, d)$ and $X_{0} \subset X$, let $x_{0}$ be the optimal value to obtain the best effect and the correlation function be

$$
k(x)=\left\{\begin{array}{r}
\frac{\rho\left(x, x_{0}, X_{0}\right)}{\rho(x, X)-\rho\left(x, X_{0}\right)}, \rho(x, X)-\rho\left(x, X_{0}\right) \neq 0 \\
\frac{\rho\left(x, x_{0}, X_{0}\right)}{a-b}, \rho(x, X)-\rho\left(x, X_{0}\right)=0
\end{array}\right.
$$

where

$$
\begin{aligned}
& \rho\left(x, X_{0}\right)=\left|x-\frac{a+b}{2}\right|-\frac{b-a}{2} \\
& \rho(x, X)=\left|x-\frac{c+d}{2}\right|-\frac{d-c}{2}
\end{aligned}
$$


If $x_{0}=a$, the left distance formula is used as:

$$
\rho\left(x, x_{0}, X_{0}\right)=\left\{\begin{array}{l}
a-x, x \leq a \\
x-b, x>a
\end{array}\right.
$$

If $x_{0}=b$, the right distance formula is used

$$
\rho\left(x, x_{0}, X_{0}\right)=\left\{\begin{array}{l}
a-x, x \leq b \\
x-b, x>b
\end{array}\right.
$$

According to the practical significance of each index, it can be divided into three types: cost type, benefit type and moderate type. For the cost index, the smaller the value is, the higher the development level of power grid is, so the left distance formula should be used. For the benefit index, the larger the value is, the higher the power grid level is, and the right distance formula should be used. For the moderate index, the development level of power grid first increases and then decreases with the increase of its value.

After obtaining the correlation matrix of each index with respect to each grade, the AHP structure is required to obtain the correlation degree of the index at the criterion level through the continuous load operation of the correlation degree of the index level and its index weight, and so on.

In this paper, AHP 1-9 scaling method is used to determine the weight vector:

$$
\boldsymbol{W}=\left[w_{1}, w_{2}, \cdots w_{n}\right]
$$

The correlation degree of criterion layer and target layer can be obtained by weighted calculation.

$$
K_{A}=W_{B} \cdot K_{B}
$$

Among them, $\boldsymbol{K}_{\boldsymbol{A}}$ represents the upper correlation degree, $\boldsymbol{K}_{\boldsymbol{B}}$ represents the lower correlation degree, and $\boldsymbol{W}_{\boldsymbol{B}}$ represents the weight vector corresponding to the lower correlation degree.

After obtaining the correlation degree of the final target layer for each level, according to the principle of maximum correlation degree, the final diagnosis level of power grid development level can be obtained, and finally the power grid development diagnosis can be realized by each index data.

\section{Case analysis and applications}

This paper uses the above basic data statistics results to verify the feasibility of the established index system and diagnostic model by taking the power grid of a province in China as an example. Each index is determined as five grades, $k_{1}, k_{2}, k_{3}, k_{4}$ and $k_{5}$, which respectively represent "excellent", "good", "medium", "qualified" and "poor".

The classical domain matter element $\boldsymbol{R}_{j}$ represents the index value range of each grade. Node domain matter element $\boldsymbol{R}_{p}$ represents the index value range of all grades. The matter element $\boldsymbol{R}$ to be evaluated represents the measurement data of each index to be evaluated, where

$$
\begin{aligned}
& R_{1}=\left[\begin{array}{ccc}
N_{1} & c_{11} & (0,2) \\
& c_{12} & (-4,2) \\
& \ldots & \ldots \\
& c_{74} & (0,0.2)
\end{array}\right], \ldots, \quad R_{5}=\left[\begin{array}{ccc}
N_{5} & c_{11} & (12,15) \\
& c_{12} & (12,15) \\
\ldots & \ldots \\
& c_{74} & (0.8,1)
\end{array}\right], \\
& R_{p}=\left[\begin{array}{ccc}
N & c_{11} & (0,15) \\
& c_{12} & (-4,15) \\
\ldots & \ldots \\
& c_{74} & (0,1)
\end{array}\right], \quad R=\left[\begin{array}{ccc}
N_{0} & c_{11} & 11.37 \\
& c_{12} & 5.34 \\
\ldots & \ldots \\
& c_{74} & 0.57
\end{array}\right] .
\end{aligned}
$$

By substituting the above three matter element models into equations (4)-(8), the correlation function values of each index with respect to different levels can be obtained.

Using AHP-1-9 scaling method, according to the expert evaluation score, the judgment matrix of index layer to criterion layer and criterion layer to target layer is given. For example, in the criterion of coordination of power grid development scale, the judgment matrix of index layer to criterion layer is as follows:

$$
R_{c_{2 n} \sim c_{2}}=\left[\begin{array}{cccc}
1 & 3 / 2 & 2 & 5 / 2 \\
2 / 3 & 1 & 3 / 2 & 2 \\
1 / 2 & 2 / 3 & 1 & 3 / 2 \\
2 / 5 & 1 / 2 & 2 / 3 & 1
\end{array}\right]
$$

The weight vector can be obtained after the single level sorting, total level sorting and consistency test. The other weights are obtained by the same method, and the results are shown in Table 1.

By substituting the results of each weight and correlation degree into equation (10), the final correlation degree of power grid development level of a province can be obtained. According to the principle of maximum correlation degree, the final diagnosis level of a province power grid development level is "medium". According to the properties of correlation function, the diagnosis results are analyzed.

The grade of each index can reflect the main problems existing in the power grid. The transformation trend of each index can provide reference for improving the development level of power grid in the future. For example, "Smart Substation proportion" and "line loss rate" belong to the "qualified" level, and the trend of transformation to "poor" level is larger than that to "medium" level. Therefore, we can focus on these two indicators and improve them. By analogy to the analysis process of a province's power grid, the development level of regional power grid can be analyzed.

\section{Conclusion}

The application of matter element analysis method in the diagnosis of power grid development can more accurately evaluate the development level of power grid, better predict the change trend of each index and the overall development situation, and provide the basis for clarifying the improvement focus and establishing the development policy. The following conclusions can be drawn. 
Table 1. Correlation function value and weight.

\begin{tabular}{|c|c|c|c|c|c|c|c|c|}
\hline Norm & Weight & Index & Weight & $k_{1}$ & $k_{2}$ & $k_{3}$ & $k_{4}$ & $k_{5}$ \\
\hline \multirow{6}{*}{$c_{1}$} & \multirow{6}{*}{0.1548} & $c_{11}$ & 0.2727 & -0.14789 & 0.79 & -0.395 & -0.59667 & -0.6975 \\
\hline & & $c_{12}$ & 0.1818 & -0.36945 & -0.14664 & 0.2925 & -0.195 & -0.39625 \\
\hline & & $c_{13}$ & 0.1818 & -0.49167 & -0.32222 & 0.016667 & -0.01613 & -0.33696 \\
\hline & & $c_{14}$ & 0.1818 & -0.455 & -0.27333 & 0.09 & -0.07627 & -0.35119 \\
\hline & & $c_{15}$ & 0.1818 & -0.49125 & -0.32167 & 0.0175 & -0.01691 & -0.33713 \\
\hline & & $c_{21}$ & 0.3859 & -0.35714 & -0.1 & 0.375 & -0.25 & -0.55 \\
\hline \multirow{3}{*}{$c_{2}$} & \multirow{3}{*}{0.1548} & $c_{22}$ & 0.2772 & 0.75 & -0.75 & -0.875 & -0.91667 & -0.9375 \\
\hline & & $c_{23}$ & 0.196 & -0.32098 & 0.057724 & -0.05175 & -0.36783 & -0.52588 \\
\hline & & $c_{24}$ & 0.1409 & -0.47429 & -0.29905 & 0.051429 & -0.04663 & -0.34403 \\
\hline \multirow[t]{3}{*}{$c_{3}$} & \multirow[t]{3}{*}{0.1548} & $c_{31}$ & 1 & -0.39357 & -0.19143 & 0.212857 & -0.1493 & -0.37018 \\
\hline & & $c_{41}$ & 0.1667 & 0.761905 & -0.7619 & -0.88095 & -0.92063 & -0.94048 \\
\hline & & $c_{42}$ & 0.1667 & 0.761905 & -0.7619 & -0.88095 & -0.92063 & -0.94048 \\
\hline \multirow[t]{5}{*}{$c_{4}$} & \multirow[t]{3}{*}{0.2209} & $c_{43}$ & 0.1111 & 0.761905 & -0.7619 & -0.88095 & -0.92063 & -0.94048 \\
\hline & & $c_{44}$ & 0.1111 & 0.761905 & -0.7619 & -0.88095 & -0.92063 & -0.94048 \\
\hline & & $c_{45}$ & 0.1111 & -0.5075 & -0.34333 & -0.015 & 0.5 & -0.32993 \\
\hline & \multirow{6}{*}{0.1049} & $c_{51}$ & 0.125 & -0.84091 & -0.78788 & -0.68182 & -0.36364 & 2.333333 \\
\hline & & $c_{52}$ & 0.1875 & -0.28986 & 0.224988 & -0.15517 & -0.43678 & -0.57758 \\
\hline \multirow{4}{*}{$c_{5}$} & & $c_{53}$ & 0.1875 & 0.952 & -0.952 & -0.976 & -0.984 & -0.988 \\
\hline & & $c_{54}$ & 0.125 & -0.31343 & 0.095238 & -0.08 & -0.38667 & -0.54 \\
\hline & & $c_{55}$ & 0.1875 & -0.15517 & 0.775 & -0.3875 & -0.59167 & -0.69375 \\
\hline & & $c_{56}$ & 0.1875 & -0.6625 & -0.55 & -0.325 & 0.35 & -0.20588 \\
\hline \multirow{2}{*}{$c_{6}$} & \multirow{2}{*}{0.1049} & $c_{61}$ & 0.4 & -0.38574 & -0.20379 & 0.656 & -0.328 & -0.552 \\
\hline & & $c_{62}$ & 0.6 & 0.029661 & -0.028 & -0.514 & -0.757 & -0.838 \\
\hline \multirow{4}{*}{$c_{7}$} & \multirow{4}{*}{0.1049} & $c_{71}$ & 0.2 & -0.45833 & -0.27778 & 0.083333 & -0.07143 & -0.35 \\
\hline & & $c_{72}$ & 0.2 & -0.44 & -0.25333 & 0.12 & -0.09677 & -0.35632 \\
\hline & & $c_{73}$ & 0.3 & -0.675 & -0.56667 & -0.35 & 0.3 & -0.1875 \\
\hline & & $c_{74}$ & 0.3 & -0.34848 & -0.06522 & 0.425 & -0.28333 & -0.4625 \\
\hline
\end{tabular}

(1) The index system reflecting the coordination of power grid development speed, power grid development scale, power grid structure, power grid security and reliability, equipment level, power grid utilization efficiency and power grid development benefit is reconstructed, and the construction principle and index selection basis are given.

(2) The matter element analysis model of power grid development diagnosis is constructed by matter element analysis method, and the correlation function is used to reflect the overall development level of power grid and the change trend of each index.

(3) The example analysis and application show that the model in this paper is able to determine the overall development level of power grid and provide the basis for the development of power grid.

\section{Acknowledgement}

This paper was supported by the science and technology project of State Grid Jiangsu Electric Power Co., Ltd. under grant J2020111.

\section{References}

1. Liu Z., Zhang Q. (2013) Research on development mode of State Grid. Journal of China Electrical Engineering, 33(07): 1-10.

2. Huang Y., Chen H. (1999) The designing methodology of extenics-based fuzzy reasoning model. IEEE SMC'99, Tokyo, Japan.

3. Liu J., Lai J., Sun X. (2015) The performance evaluation of construction project management model based on matter element analysis method. Journal of Engineering Management, 1: 124-128.

4. Huang H., Luo W., Wu C., et al. (2010) Evaluation of land ecological security based on matter element analysis. Journal of Agricul. Eng., 26: 316-322.

5. Wang S., Zhao D. (2020) A hierarchical power grid fault diagnosis method using multi-source information. IEEE Transactions on Smart Grid, 11(3): 2067-2079.

6. He Y., Zheng X., Tai N., et al. (2020) A DC line protection scheme for MMC-based DC grids based 
on AC/DC transient information. IEEE Trans.

Power Deli.,35(6): 2800-2811. 